\title{
Product Failure Time Assessments using Early Degradation Filtering
}

\author{
Emmanuel Bender* and Joseph B Bernstein \\ Department of Microelectronics Reliability, Ariel University, Israel
}

Submission: May 28, 2021; Published: June 09, 2021

"Corresponding author: Emmanuel Bender, Department of Microelectronics Reliability, Ariel University, Ariel, Israel

\begin{abstract}
Here, we demonstrate a novel procedure for generating reliability evaluations in device aging tests run very close to operation conditions. Packaged devices are aged in multiple temperature and frequency modes. Device elements that show early degradation are isolated to generate accurate mean failure times. A correlation between ring oscillator (RO) circuit size and $\beta$ slopes of Weibull failure distributions are used to indicate the confidence level of the data. Via shaping of the distributions, the aging information is successfully separated from the system noise. The technique is proven to be effective for device reliability testing and suitable for device health monitoring.
\end{abstract}

Keywords: FPGA; RO; FinFET; BTI; Weibull failure distributions

\section{Introduction}

The formula for success in microelectronic reliability testing is to deliver precise failure estimations within time-to-market constraints [1]. To obtain results quickly, tests are accelerated by means of stressors such as voltage, current and temperature. In testing methods such as the High-temperature operating life (HTOL) method, the stress levels induced are far beyond operation conditions [2]. Results are received quickly, but the credibility of the results are low because of concern of physical variation of the device due to the stress. Alternatively, low stress tests must be very long to receive results discernible from noise.

In this study, a novel technique for low stress testing is proposed based on the Multi-Temperature Operational Life (MTOL) testing method [3]. The method is implemented with 200 hour device aging tests using variable voltage frequency and temperature stress conditions. Here, stress conditions are chosen that virtually match operation conditions. The objective is to show that sufficient degradation data is received to generate an accurate reliability assessment.

FPGA devices are programmed with multi-sized RO circuits to provide degradation data on a large range of frequencies. The results received allow the separation of failure mechanisms: BTI, EM and HCI as is detailed in previous studies [4,5].

Application of the MTOL method on low stress tests successfully generates lifetime data congruent with data received from tests in extreme stress conditions. Such a procedure is advantageous in reliability testing because it ensures preservation of the physical characteristics of the device. The methodology can also be principle in designing an embedded sensory module because it is effective in regular use conditions.

\section{Experimental Results}

A series of tests with variable temperature stresses and a nominal voltage of $0.85 \mathrm{~V}$ were run on Ultra96 boards using $16 \mathrm{~nm}$ FinFET technology [6]. Since the chip was programmed with rings of variable sizes, data was generated over a large range of frequencies. Device failure times were obtained by finding the slope of the degradation using the appropriate time power law and extrapolating to a performance decrease of $10 \%$. The data acquired in variable stress modes and frequencies provided an ideal basis for revealing and separating the dominant failure mechanisms in the chip.

We demonstrated in previous studies that the amount of dispersion of the time-to-failure (TTF) values directly corresponds to number of stages in the ring circuits [7]. When the TTF values are set to a ring frequency plot, the data in low frequencies conserves a tight pattern and fans out in the higher frequency rings (Figure 1). In any case, even the small rings with the most dispersed TTF values retain a high level of determinism. The interaction between the stages in a ring cause the circuit to show characteristics of a parallel failure system. The propagation delay of the stages averages together to generate the frequency 
of the ring. As the size of the ring increases, the TTF value of the circuit becomes more precise based on the central limit theorem. The Weibull distribution is commonly used for analysing diverse types of aging phenomenon (Eq. 1 below).

$$
R(t)=e^{-(t / \theta)^{\beta}}
$$

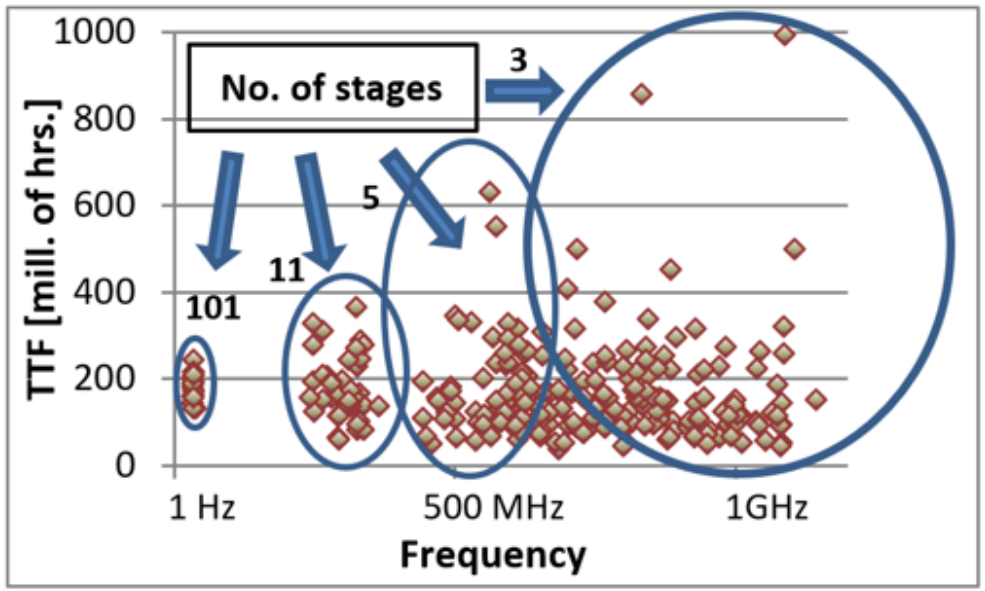

Figure 1: An example of a TTF to frequency plot. The frequency varies with number of stages in the rings (listed in the figure). The TTF values become more dispersed with increase in frequency.

Figure 2 is a collection of Weibull distributions of 3, 5, and 11 rings. Each plot consists of the TTF values of many same-sized RO circuits running in parallel. The gradient of the $\beta$ slope shows the level of determinism of the of the failure times in a Weibull distribution. A clear one-to-one correlation between the number of stages in the rings to the $\beta$ slope appears. Comparable results formulate for different stress modes and technologies.
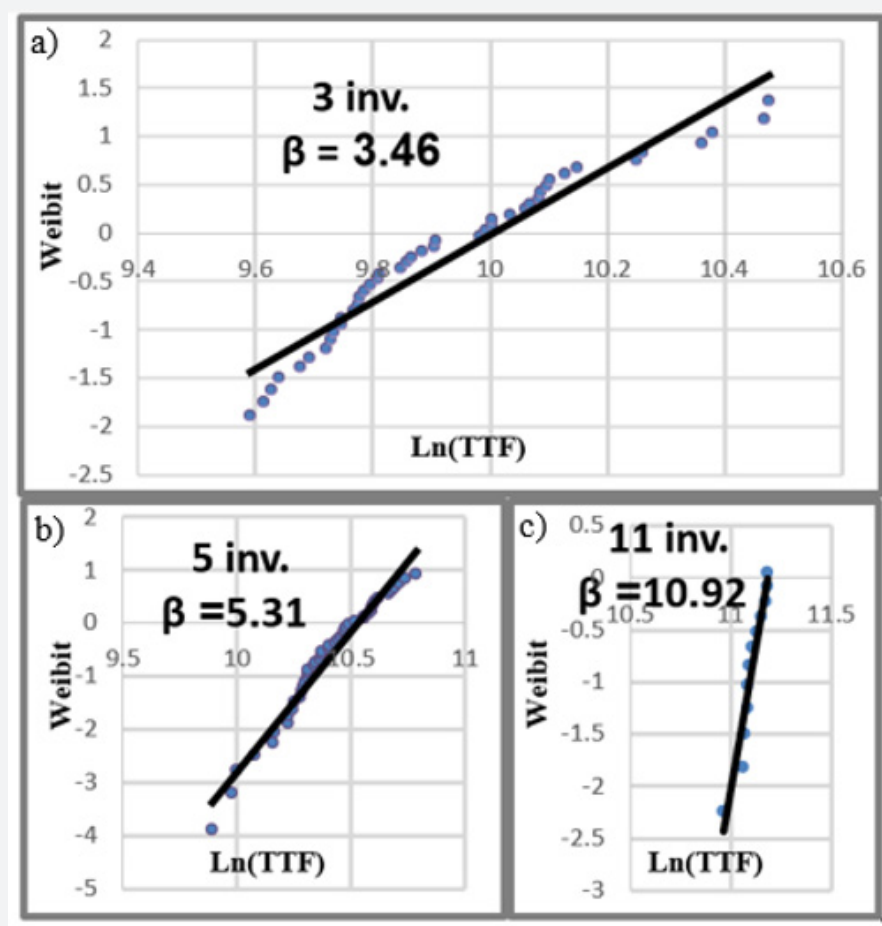

Figure 2: A set of Weibull failure distribution plots of RO circuits categorized by number of stages: 3 inverters (a), 5 inverters (b) 11 inverters (c). The $\beta$ slope is congruent with the number of stages.

When we analysed the data of the low stress tests, we received unexpected results. Neither did the data fit to a Weibull distribution nor the slope resemble that of other tests. The data also did not fit to a lognormal distribution implying that the data 
is not random. Figure 3 displays a Weibull distribution of 3 stage rings from a test at an ambient temperature of $50^{\circ} \mathrm{C}$. The plot has a distinct transition in the place where two symbolic lines (highlighted in the figure) cross. When the portion of the plot marked by the yellow line is measured separately on a Weibull distribution, a $\beta$ slope of 3.004 is received. When the data hooking to the right is formulated into a separate Weibull distribution, it has a slope below one $(\beta<1)$ suggesting totally random events. Such a distribution would be expected for noise.

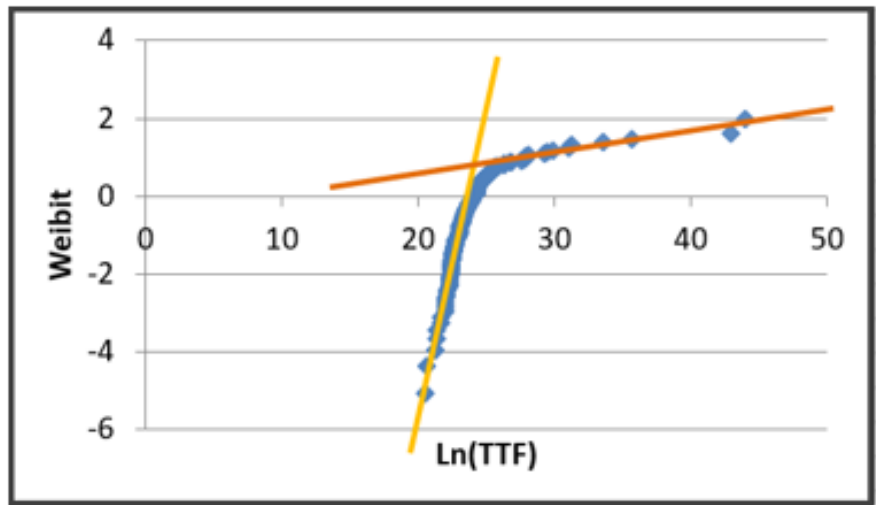

Figure 3: A Weibull plot of 3 stage $\mathrm{RO}$ in an ambient temperature of $50^{\circ} \mathrm{C}$ and $0.85 \mathrm{~V}$. In the plot, there is a transition marked with two lines (yellow and orange). The transition indicates a mixture of failure degradation in low TTFs and noise in high TTFs.

Since the tests are under low stress, the outcome is easily explained. The TTF is figured from the slope of frequency degradation. When the slope is too slight, the degradation signal is indiscernible from the noise of the system. Figure 4 explains the data of the tests. The rate of degradation for each element is random. The early failures produce steeper slopes which will create the right side of the bell "Slope of signal" distribution. As the slopes ease off, they will mix with the "Slope of noise" distribution. Therefore, a process for filtering the data is to calculate the early failure times until the point of transition. The data beyond this point does not contribute information to the failure trend.

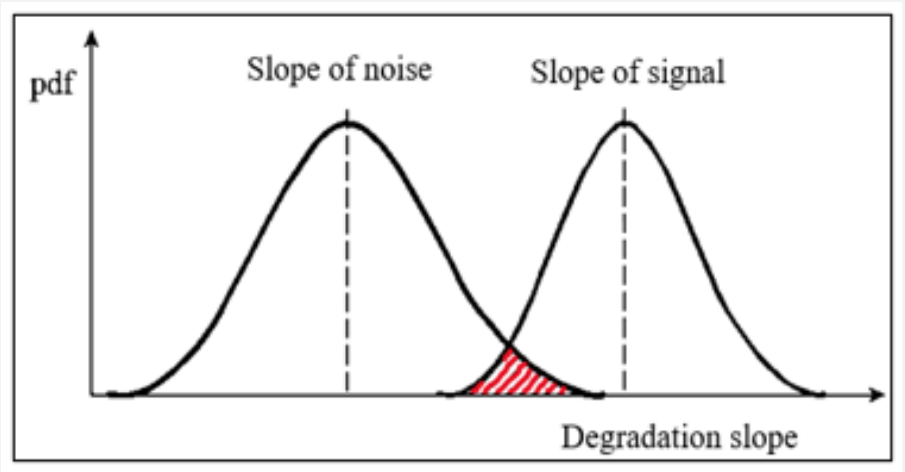

Figure 4: A depiction of mixing of slope distributions. The left is a distribution of slopes formed from system noise. The right is that of the degradation. Mixture will occur between them in low stress tests.

We found that the Weibull slopes of the early failure data are comparable to those calculated from high stress tests. This validates that the early failures are an authentic part of the signal. Basing a failure trend from the early failures is acceptable since the Weibull distribution is a weakest-link type distribution. The early failures are a prediction of the later failures [8]. Therefore, it is sufficient to observe the early failures to see the trend of the system. The mean time-to-fail (MTTF) value or $\theta$ is the zero point of the Weibull plot. Since we have actual TTF measurements with their Weibull characteristics, each test reveals the reliability distribution function of the device for that specific stress condition.

The results of 3 tests in ambient temperatures of $30^{\circ} \mathrm{C}, 50^{\circ} \mathrm{C}$, and $70^{\circ} \mathrm{C}$ are analysed in 3 different frequency ranges, from $250 \mathrm{MHz}$ up to $1 \mathrm{GHz}$. The ranges of the frequencies are chosen by number 
of stages in the circuit: 3,5 , and 11 . The results of the analysis are displayed in Figure 5 on the next page. The plots show a distinct transition between higher stress results compared to lower stress results. Highest frequency and highest ambient temperature test is shown in $5 \mathrm{c}$. In this plot, there is a characteristic Weibull distribution for all the data. As the stress diminishes, the left direction for frequency decrease ( $5 a$ and $5 b$ ) and downward for temperature decrease ( $5 \mathrm{f}$ and $5 \mathrm{i}$ ), there is a consistent increase in distortion to the Weibull slope. This is visible both in the shape of the plot and clear from the $\beta$ slope which decreases continuously. The percentage of data used to find the $\beta$ slope is listed in each plot. As the stress decreases, both the percentage used and the precision decreases. This fortifies the premise mentioned earlier that, as the stress of the test is lowered, the noise in the results is more visible in the data. Correction of the data by excluding longer failure times is less effective with the decrease in stress. In any case, the results obtained by filtering out non-fitting parts of the plot show a high level of accuracy. The outcome shows frequency dependence as is seen that the MTTF is shorter in the higher frequencies. The results of the test well within the range of values received in the high temperature voltage and frequency stress tests run in previous studies [5].

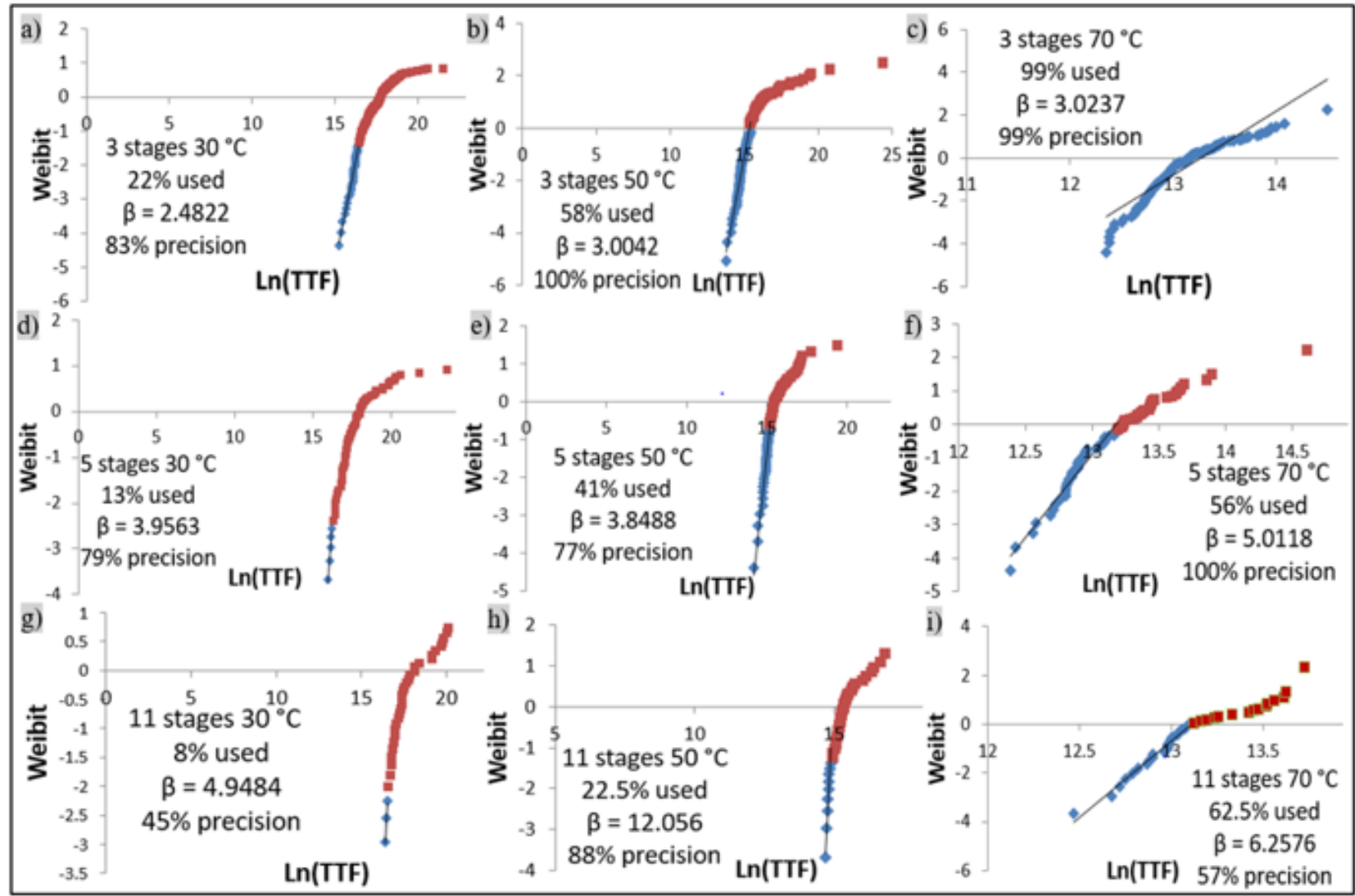

Figure 5: A collection of Weibull distribution plots summarizing the inspection of low stress testing on the device. The plots show a degression of failure signal as the stress decreases. $5 \mathrm{a}-5 \mathrm{c}$ are tested at $30^{\circ} \mathrm{C} .5 \mathrm{~d}-5 \mathrm{f}$ are tested at $50^{\circ} \mathrm{C} .5 \mathrm{~g}-5 \mathrm{i}$ are tested at $70^{\circ} \mathrm{C}$. The three different plots in each temperature represents a different frequency mode. The top row, made of 3 rings, is about $1 \mathrm{GHz}$. The middle row, made of 5 rings, is about $600 \mathrm{MHz}$. The bottom row, made of 11 rings, is about $250 \mathrm{MHz}$.

\section{Conclusion}

We have demonstrated how it is possible to generate failure times from reliability testing that is virtually at operation conditions. We succeeded in producing failure data that, is discernable although there is a non-negligible amount of noise. By use of a novel filtering procedure, we succeeded in filtering the failure data from the noise using Weibull distribution analysis. The results are congruent with testing operated in higher stress. This verifies that the method is effective in these low stress conditions.

\section{Acknowledgment}

Many thanks for the on-going support from: Ariel University Advanced Studies Research Group, Ariel Israel.

\section{References}

1. G Delarozee (1999) Introduction to Reliability. Microelectronic Engineering 49(1-2): 3-10.

2. Xilinx (2015) Device Reliability Report, UG116 (v10.3.1). 
3. JB Bernstein, M Gabbay, O Delly (2014) Reliability matrix solution to multiple mechanism prediction. Microelectron Reliab 54(12): 29512955.

4. JB Bernstein, A Bensoussan, E Bender (2017) Reliability prediction with MTOL. Microelectron Reliab 68: 91-97.

5. JB Bernstein, A Bensoussan, E Bender (2020) Reliability Prediction of FinFET FPGAs by MTOL. Microelectron Reliab 114: 113809.
6. (2018) Ultra96 Hardware User's Guide, Version 0.9, Avnet Inc.

7. E Bender, JB Bernstein (2021) Microchip Health Monitoring System Using the FLL Circuit. Sensors 21(7): 2285.

8. MP McPheirson (2010) Reliability and Physics Engineering. Springer.

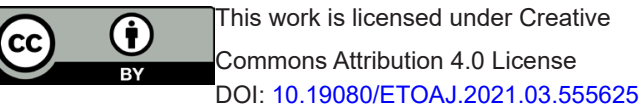

\author{
Your next submission with Juniper Publishers \\ will reach you the below assets \\ - Quality Editorial service \\ - Swift Peer Review \\ - Reprints availability \\ - E-prints Service \\ - Manuscript Podcast for convenient understanding \\ - Global attainment for your research \\ - Manuscript accessibility in different formats \\ ( Pdf, E-pub, Full Text, Audio)
}

- Unceasing customer service

Track the below URL for one-step submission https://juniperpublishers.com/online-submission.php 